\title{
MITE-transposon Display Efficiently Detects Polymorphisms among the Oryza
} AA-genome Species

\author{
Kyoko Takagi, Hironori Nagano, Yuji Kishima* and Yoshio Sano \\ Laboratory of Plant Breeding, Faculty of Agriculture, Hokkaido University, Kita-ku Kita 9 Nishi 9, Sapporo, Hokkaido 060-8589, Japan
}

\begin{abstract}
Miniature inverted repeat transposable elements (MITEs) are a major component of interspersed repetitive sequences in the rice genome. These elements serve as excellent tools for fine genomic analysis throughout the genome. Using the rice genome database, we evaluated MITEs present in a $200-\mathrm{kb}$ region surrounding the rice waxy locus and selected four MITE subfamilies for MITE-transposon display (MITE-TD) analyses. MITETD, which is an AFLP-related technique based on MITE sequences, was applied to detect polymorphisms among the AA-genome Oryza species using the four selected MITEs. The MITE-TD used here enabled the most efficient detection of polymorphisms of all the molecular marker techniques applied to date in the Oryza species. Of the four MITEs, Mashu, a new MITE family, was found to be the best system for detecting the polymorphisms, with a detection frequency 3-1.5 times higher than that of the other three MITEs. The MITETDs also revealed information about genetic variations within the AA-genome species, and the complexity of the genetic relationships between $O$. sativa and o. rufipogon.
\end{abstract}

Key Words: Oryza species, MITE, transposon display, phylogenetic tree.

\section{Introduction}

The grass species vary in genome size (Bennetzen 2000). The larger-genome species of grasses such as maize and barley arose via a massive amplification of class 1 type elements, long terminal repeat (LTR) retrotransposons (SanMiguel et al. 1996, Suoniemi et al. 1996). Inevitably, the amplification of LTR retrotransposons resulted in expansion of the spacer regions in those grass species (Bennetzen et al. 1998, Kumar and Bennetzen 1999). On the other hand, the small genome species such as rice do not successfully amplify LTR retrotransposons, and thus they contain small spacers (Tarchini et al. 2000, Dubcovsky et al. 2001). However, abundant miniature inverted repeat transposable elements (MITEs) have been found in the spacer regions of the rice

Communicated by N. Kurata

Received October 26, 2002. Accepted January 10, 2003.

*Corresponding author (e-mail: kishima@abs.agr.hokudai.ac.jp) genome (Bureau and Wessler 1994a, 1994b, Lisch et al. 2001, Nagano et al. 2002). MITE is a collective name for small transposon-like elements with terminal inverted repeats (TIRs) at the ends (Wessler et al. 1995). MITE families have been found in plants and animals, and most seem to be inactive (Feschotte et al. 2002). In contrast with active class 2 type elements, MITEs are characterized by uniformly small sizes $(<500 \mathrm{bp})$ and by target site preferences (Feschotte et al. 2002). MITEs also display a very high copy number and an even distribution throughout the chromosomes (Casa et al. 2000). They are known to be major elements contributing to the repetition in the rice genome architecture (Bureau et al. 1996, Turcotte et al. 2001, Nagano et al. 2002). However, little is known about the relative distribution and dynamism of MITEs among various rice species. If MITEs can be inserted into species-specific unique sites, they would provide a useful tool for characterizing genetic relationships at the whole-genome level.

In this study, we used the transposon-display (TD) method for MITEs-mediated phylogenetic analysis among rice-related species (Van den Broeck et al. 1998, Casa et al. 2000). We characterized repetitive sequences in a $200-\mathrm{kb}$ region around the rice waxy locus, and identified 55 transposable element (TE)-like sequences (Nagano et al. 2002) therein. Fifty of the 55 TE-like sequences consisted of MITEs or MITE-like sequences, most of which are categorized into three representative MITE families, Stowaway, Tourist and $\mathrm{Mu}$-like families (the Mu-like family is usually distinguishable from MITEs, since this family normally consists of copies larger than $500 \mathrm{bp}$ ). Here we studied major subfamilies with an abundant copy number predicted in the rice genome database from each of the three representative MITE families. In addition, one element from a newly identified MITE family around the rice waxy locus was also studied. The MITEs examined here were expected to become useful molecular markers within the AA-genome Oryza species from now on. Among the four MITE subfamilies selected for this study, Mashu, the new MITE subfamily, was found to have a markedly conserved copy organization, and to create polymorphisms in the AA-genome species at high frequency, suggesting that it has been active until recently. The phylogenetic study via MITE-TD showed that O. rufipogon possesses a wide range of genetic variation, suggesting its association with the cultivated rice species. 


\section{Materials and Methods}

Plant materials and genomic DNA extraction

Twenty-one lines from six Oryza species with the AAgenome and $O$. officinalis with the $\mathrm{CC}$-genome were used in these experiments (Table 1). They were maintained as inbred lines by several selfings, except for $O$. officinalis. Total genomic DNAs were isolated as described by Nagano et al. (1999).

\section{MITE-TD}

The TD method used here was basically similar to the method described by Casa et al. (2000). We targeted four MITE elements: Stowaway OS-1 in the Stowaway/Tnr1 family, Tabito II in the Tourist family, Onaga I in the Mulike family and Mashu in the Mashu family (Nagano et al. 2002). All the primers used here are listed in Table 2. The $T a q \mathrm{I}$ adaptor was prepared by annealing the two adaptor primers at room temperature after boiling. The TaqI adaptor
$(50 \mu \mathrm{M})$ was ligated to the TaqI-digested genomic DNA (200 ng) by adding a mix containing $10 \mathrm{X}$ ligation buffer, 0.5 $\mu \mathrm{M}$ ATP and 1 Weiss unit of T4 DNA ligase and incubated at $16^{\circ} \mathrm{C}$ overnight. Each of the primary primer sets was designed from the adaptor sequence and the terminal sequences of each MITE, and the first PCR reaction was performed in $20 \mu \mathrm{l}$ of reaction mixture containing 1 X PCR buffer, $0.2 \mathrm{mM}$ dNTPs (Takara Shuzo Co Ltd., Osaka), 15 ng of the adaptor-ligated DNA, 10 pmol of each primary primer and 1 unit of EX Taq polymerase (Takara Shuzo Co Ltd., Osaka). The first PCR reaction was carried out as follows: $72^{\circ} \mathrm{C} / 2 \mathrm{~min} ; 94^{\circ} \mathrm{C} / 3 \mathrm{~min} ; 24 \mathrm{cycles}$ of $94^{\circ} \mathrm{C} / 30 \mathrm{sec}$, $54^{\circ} \mathrm{C} / 30 \mathrm{sec}$ and $72^{\circ} \mathrm{C} / 1 \mathrm{~min}$, and a final cycle of $72^{\circ} \mathrm{C} / 5 \mathrm{~min}$. The PCR product was then diluted 20 -fold with sterilized water.

Nested primers were used for the secondary PCR: $5^{\prime}$ end of the secondary MITE primers were labeled with FITC, and the $3^{\prime}$ end of the secondary adaptor primers were overhung by three selective nucleotides (in this study, we ran-

Table 1. A list of the Oryza strains used in this study

\begin{tabular}{|c|c|c|c|c|}
\hline Genome & Oryza species & Type & Strain & Origin \\
\hline \multirow[t]{20}{*}{ AA } & O.sativa & Japonica & A58 & Japan \\
\hline & & & Koshihikari & Japan \\
\hline & & & T65 & Taiwan \\
\hline & & Javanica & 221 & Philippines \\
\hline & & & 627 & Celebes Is. \\
\hline & & Indica & IR36 & Philippines \\
\hline & & & Kasalath & India \\
\hline & & & 414 & India \\
\hline & & & 868 & Taiwan \\
\hline & O.rufipogon & annual & W107 & India \\
\hline & & & W2002 & India \\
\hline & & & W630 & Myanmar \\
\hline & & & W1551 & Thailand \\
\hline & & perennial & W120 & India \\
\hline & & & W149 & India \\
\hline & & & W1718 & China \\
\hline & O.glaberrima & & W025 & Guinea \\
\hline & O.barthii & & W1579b-2 & Cameroun \\
\hline & O.glumaepatula & & W1185 & Surinam \\
\hline & O.meridionalis & & W1625 & Australia \\
\hline $\mathrm{CC}$ & O.officinalis & & W0002 & Unknown \\
\hline
\end{tabular}

Table 2. Oligonucleotide sequences used for adaptors, adaptor primers and MITE primers

\begin{tabular}{lll}
\hline \hline Type & Name & Sequence $\left(5^{\prime} \rightarrow 3^{\prime}\right)$ \\
\hline Adaptor & & GTGCTTGATGCTTGAAAC+CGGTTTCAAGCATCAA \\
Adaptor primer & adaptor 1st & GTGCTTGATGCTTGAAACCGA \\
& adaptor 2nd & GTGCTTGATGCTTGAAACCGANNN \\
MITE primer & Stowaway Os-1 1st & GAATGACTTACATTGTGAAACGGAG \\
& Stowaway Os-1 2nd & GACTTACATTGTGAAACGGAGGGAG \\
& Tabito II 1st & GATGGAAAAGTTGRAAGTTTGAAGAAAAAG \\
& Tabito II 2nd & TTGAAGAAAAAGTWGGAACTAAACWCGGC \\
& Onaga I $1 \mathrm{st}$ & AATCGGATTCGTTGACAAGTTAAGG \\
& Onaga I 2nd & CGTTGACAAGTTAAGGGACCTYAGAT \\
& Mashu 1st & ATAGATAGCACCTTACTTTACCATTG \\
& Mashu 2nd & CCTTACTTTACCATTGYGGGTGCTC \\
\hline
\end{tabular}


domly selected three kinds of secondary adaptor primers for each MITE-TD). The second PCR reaction was performed with the same reaction mixture as the first PCR plus $2 \mu \mathrm{l}$ of $50 \%$ DMSO. For the temperature cycling, we used a modification of the "touchdown method": $94^{\circ} \mathrm{C} / 5 \mathrm{~min}$, then 10 cycles beginning with $94^{\circ} \mathrm{C} / 30 \mathrm{sec}, 68^{\circ} \mathrm{C} / 30 \mathrm{sec}, 72^{\circ} \mathrm{C} / 1 \mathrm{~min}$, followed by a reduction of the annealing temperature of the first cycle $\left(68^{\circ} \mathrm{C}\right)$ in $1{ }^{\circ} \mathrm{C}$ increments per cycle, and then 27 cycles at an annealing temperature of $58^{\circ} \mathrm{C}$, and a final incubation at $72^{\circ} \mathrm{C} / 5 \mathrm{~min}$.

The reaction products were electrophoresed on a $3.5 \%$ denaturing polyacrylamide gel containing $50 \mathrm{ml}$ of a mixture of $3.5 \mathrm{ml}$ of Long Ranger (equivalent to $50 \%$ polyacrylamide : bisacrylamide solution $=19: 1$ ) and $7.0 \mathrm{M}$ urea. After the gel was pre-run using $1 \mathrm{X}$ TBE as running buffer, the samples were electrophoresed at a constant $1700 \mathrm{~V}$ for 80 $\min$. The bands were visualized by scanning the fluorescent signals in the gel plate with a Typhoon 8600 (Molecular Dynamics Inc. Sunnyvale, California).

\section{Data analysis}

After the scan, the visualized gel profiles were saved as an ImageQuant image file (Molecular Dynamics Inc. Sunnyvale, California), and the sizes of the bands on the image were characterized by Fragment Analysis (Molecular Dynamics Inc. Sunnyvale, California). Each informative band was scored independently as 1 for "presence" and 0 for "absence". To estimate the level of polymorphisms between a pair of lines, the similarity coefficient (SC) (Dice 1945) between a pair of entries was calculated as

$$
\mathrm{SC}=\mathrm{N} /(\mathrm{Ni}+\mathrm{Nj}-\mathrm{N})
$$

where $\mathrm{N}$ is the number of shared fragments and $\mathrm{Ni}$ and $\mathrm{Nj}$ are the total numbers of fragments for entries $\mathrm{i}$ and $\mathrm{j}$ (Lynch 1990, Zhou and Gustafson 1995). The genetic distance coefficient (GDC) was calculated as

$$
\mathrm{GDC}=1-2 \mathrm{~N} /(\mathrm{Ni}+\mathrm{Nj})
$$

for constructing the phylogenetic trees. We constructed a neighbor-joining (NJ) tree based on these estimates (Saitou and Nei 1987). The tree was drawn using PAUP*4.0 (Swofford 1998).

\section{Results and Discussion}

\section{Selection of MITEs for genome survey}

A high copy number, conserved structure and unique insertion sites are the essential features of MITEs for use as efficient DNA markers. We searched for such MITEs by selecting elements with a high copy number residing in a 200$\mathrm{kb}$ region around the $w x$ locus in the Oryza sativa genome (Nagano et al. 1999, 2000). MITEs are major components of the repetitive sequences in the rice genome, particularly of the type of class II elements in the Tourist, Stowaway/Tnr1 and $\mathrm{Mu}$-like families, which are frequently found in the rice genome. Each of these three families comprises several subfamilies (Nagano et al. 2002). The standard sequence of each subfamily was determined as the consensus of at least
10 independent sequences with over $95 \%$ homology. We performed a BLAST search (version 2.0, http://www.ncbi. nlm.nih.gov/blast/) against the rice genome sequences available as of July 2002 . Using $50 \mathrm{bp}$ of the $5^{\prime}$ end region of each element as the query, we counted the number of rice genomic segments containing sequences resulting in a score of more than 50 bits (the maximum score is 100 bits) (Table 3 ). These outcomes indicate the numbers of hit segments deposited in the rice genome database, not the number of sequences present in the rice genome database. Therefore, these numbers express the degree of frequency of the elements in the rice genome. The numbers of the hit segments ( $>50$ bits) varied among the subfamily elements. We selected subfamilies with the highest or second-highest score among each of the three families: Tabito II (Tourist family), Stowaway OS-1 (Stowaway/Tnr1 family) and Onaga I (Mu-like family) obtained 398, 551 and 216 hit numbers, respectively. Moreover, a similar search was performed using five new MITEs. Among the five new elements, Mashu was found to be the most abundant (171 hit numbers).

\section{Characteristics of the selected MITEs}

We next investigated the characteristics of the selected MITE subfamilies, focusing on the degree of genetic divergence within these families. The segments listed in Table 3 are mostly large genomic fragments $(>100 \mathrm{~kb})$, which were generally analyzed for the rice genome project, so that many segments contained several sequences homologous to the queries. For each segment, we counted the total number of sequences that had $>50$ bits against the $50 \mathrm{bp}$ of the $5^{\prime}$ ends of the four standard MITEs. The sequences that scored more than 50 bits for the query of Stowaway Os-1, Tabito II, Onaga I and Mashu hit 1454, 764, 419 and 251 sequences, respectively. Since Stowaway OS-1 and Onaga I have long TIRs, $70 \mathrm{bp}$ and $297 \mathrm{bp}$, respectively, the $50 \mathrm{bp}$ from their $5^{\prime}$ ends include TIRs. By replacing the sequence number by the copy number, we could roughly estimate the copy number per segment in each MITE as 1.3 (Stowaway Os-1), 1.0 (Onaga I), 1.9 (Tabito II) and 1.5 (Mashu). These results suggest that Tabito II shows a preference for localizing near another Tabito II element, as Jiang and Wessler (2001) reported, while the other elements show a relatively weaker insertion preference.

Fig. 1 shows the distributions of the bit scores and the percentages of the sequences hit by each MITE query in the BLAST analysis. A score of 100 bits indicates $100 \%$ homology with the $50 \mathrm{bp}$ of the $5^{\prime}$ ends of the standard sequences (but note that 50 bits is not equal to $50 \%$ homology). The majority of the copies belonging to Stowaway OS-1 and Tabito II were distributed in the low score range, and the ratio was reduced as the score increased. This tendency reflects a diverged structural organization of the two MITE subfamilies. Onaga I had a maximum range of 70-61 bits, which indicates a rather small degree of divergence among the copies. For Mashu, as many as $60 \%$ of the total number of sequences were in the range of 100-81 bits, indicating that 
Table 3. The MITE subfamilies found around the rice waxy locus and the number of segments in the rice genome database obtained by BLAST search

\begin{tabular}{|c|c|c|c|c|c|c|c|}
\hline Tourist & No. segments ${ }^{1)}$ & Stowaway/Tnr1 & No. segments & Mu-like & No. segments & New MITEs & No. segments \\
\hline TouristI & 161 & Tnr1 & 445 & $\mathrm{RMu}-\mathrm{A} 23$ & 15 & Akan & 27 \\
\hline Castaway & 105 & StowawayOs12) & 551 & Mermite & 41 & Mashu $^{2)}$ & 171 \\
\hline Ditto/TypeF 3 ) & 454 & StowawayOs4 & 12 & OnagaI $^{2)}$ & 216 & Saroma & 37 \\
\hline Gaijin & 192 & TypeA & 35 & OnagaII & 125 & Shikotsu & 53 \\
\hline TypeC & 4 & TypeB & 198 & & & Toya & 13 \\
\hline TypeE & 191 & KiseruI & 213 & & & & \\
\hline TabitoI & 31 & KiseruII & 163 & & & & \\
\hline TabitoII ${ }^{2)}$ & 398 & KiseruIII & 16 & & & & \\
\hline TabitoIII & 17 & KiseruIV & 345 & & & & \\
\hline TabitoIV & 133 & & & & & & \\
\hline TabitoV & 104 & & & & & & \\
\hline TabitoVI & 172 & & & & & & \\
\hline
\end{tabular}

All the subfamilies are categorized into the three representative MITE families and the new families.

These subfamilies are taken from Nagano et al. (2002).

1) No. segments means the number of segments with over 50 bits hit by BLAST analysis (http://www.ncbi.nlm.nih.gov/blast)).

2) The subfamilies with double asterisks were used for further analysis.

3) Ditto/TypeF had the largest number of segments hit with BLAST analysis, but we failed to design an appropriate primer for it for TD.

these were well-conserved elements among the four MITEs. The different patterns of divergence of the four MITEs lead us to suppose that the evolutionary times when they had activities in the Oryza genome differed in order of: Tabito II, Stowaway OS-1, Onaga I and Mashu (early to late). Tabito II is thought to have become inactive long before the other MITEs, because there are no sequences matching with 100 91 bits. On the other hand, more than $15 \%$ copies of Mashu correspond to 100-91 bits. Thus, it is possible that Mashu is an active element in the rice genome if its autonomous element is present.

\section{MITE-TD analysis of the Oryza AA-genome species}

We performed MITE-TD with the four selected subfamilies against 21 lines of Oryza. Appropriate primers were designed for each element and from the adaptors carrying three additional nucleotides as described in Materials and Methods. We carried out three displays on each of the MITE subfamilies with different adaptor primers. The plant materials subjected to MITE-TD have been maintained by selfing for several generations; thus, most loci are considered to have become homozygous. Table 4 summarizes the average numbers of bands detected with a single set of adaptor primers estimated from the total band numbers of MITE-TDs with the three adaptor primers. The same MITE primer resulted in nearly the same number of bands in 20 lines of the AA-genome species (Table 4). The consistent number of bands for each MITE within the AA-genome species implies that these MITEs were in the ancestral genome of the AAgenome species, and that these MITEs have not been amplified in a species-specific manner. Compared with the AAgenome species, the CC-genome species, O. officinalis, showed markedly fewer detectable bands in Stowaway OS1, Tabito II and Mashu. In the AA-genome, the fact that four MITEs had saturated copy numbers might indicate that the elements are in an inactive state.

MITE-TD readily revealed polymorphisms among the 20 lines of Oryza AA-species. The data extracted from 9 lines of $O$. sativa are summarized in Table 5. Hitherto, amplified fragment length polymorphism (AFLP) analysis showed the best performance for producing polymorphic bands in the Oryza species (Maheswaran et al. 1997, Zhu et al. 1998, Aggarwal et al. 1999). For instance, Maheswaran et al. (1997) reported that AFLP detected an average of 47.3 bands using a single primer pair and 10.4 bands $(21.8 \%$ of total number of bands) as polymorphism between IR64 (Indica) and Azucena (Japonica). The results of the MITE-TD analyses performed here revealed that each of the four trials was superior to AFLP analysis in terms of both total band numbers and frequency of occurrence of the polymorphisms between Indica and Japonica. In particular, Mashu generated polymorphisms at frequencies of 3-1.5 times higher than the other elements in most of the plots (Table 5). The copies of Mashu tend to reside in unique sites, suggesting that Mashu became active recently.

\section{Phylogenetic trees produced with MITE-TD}

Based on the polymorphisms detected by MITE-TD, we constructed phylogenetic trees for the 21 Oryza strains (Fig. 2). The four trees made for individual MITE-TDs were constructed by the NJ method. The four dendrograms show basically similar topologies for the 21 lines from the seven Oryza species, but each tree revealed differences in the relative times at which the four MITEs had been active (Fig. 2). The trees reveal that the nine strains of $O$. sativa are divided into two clusters: one includes Japonica and Javanica strains and the other includes Indica strains. O. rufipogon contributes to both of the clusters as the wild progenitor. A perennial strain of $O$. rufipogon from China (W1718) seems to be included in the Japonica and Javanica cluster, whereas the 

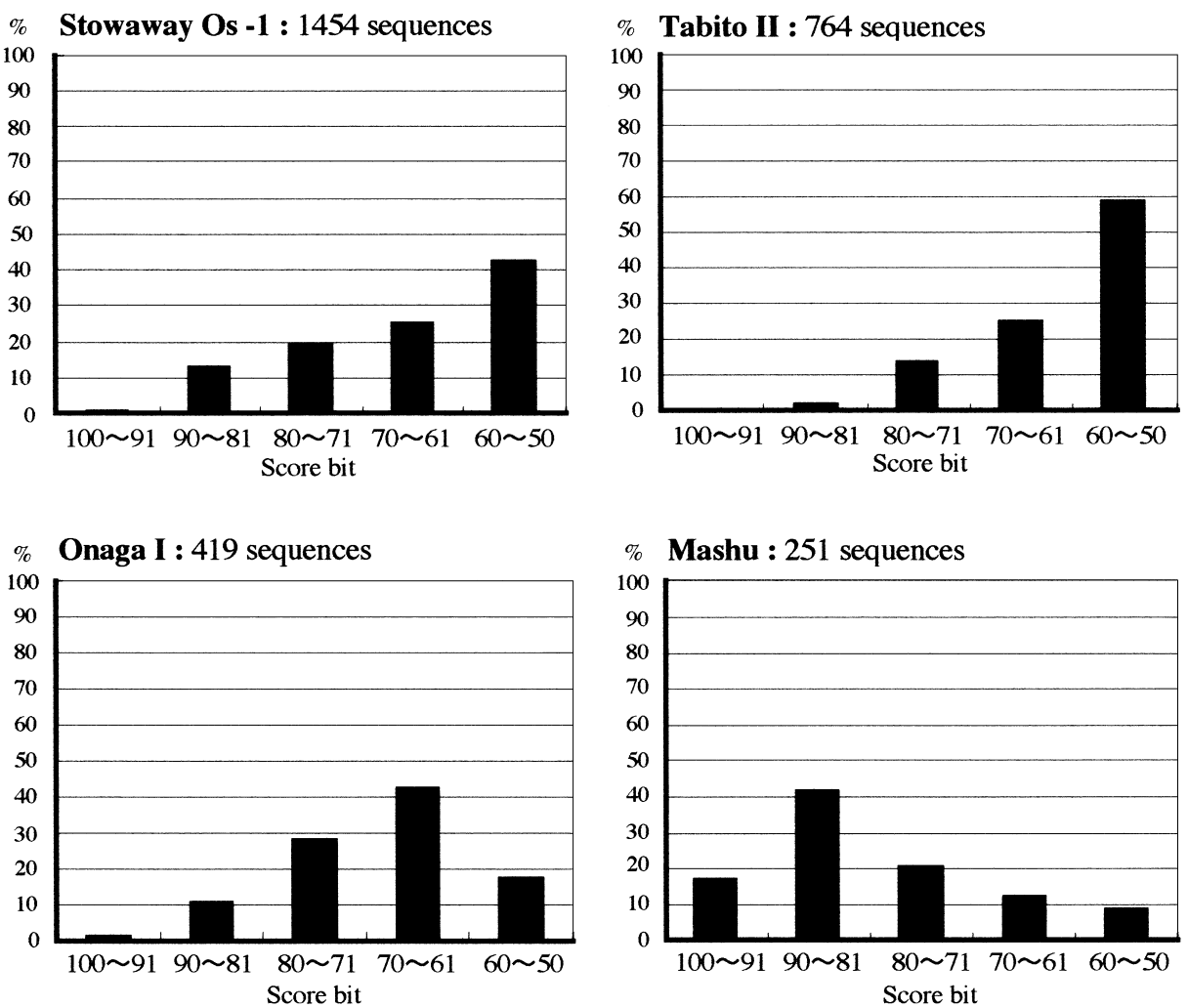

Fig. 1. Distributions according to their scores of MITE sequences as estimated by BLAST analysis against the rice genome.

Each $50 \mathrm{bp}$ from the $5^{\prime}$ end of the four MITEs was subjected to BLAST analysis (http:// www.ncbi.nlm.nih.gov/blast/). The outcome sequences with more than 50 bits obtained by the BLAST search were sorted out every 10 bits. In the case of $100 \%$ matching with the 50 -bp query, the score corresponds to 100 bits. The number of the outcome sequences obtained by the BLAST search is indicated above the histograms.

Table 4. Number of bands detected by the four MITE-TDs in the 21 Oryza strains ${ }^{1), 2)}$

$\mathrm{AA}$

MITE

A58 $\begin{gathered}\text { Koshi- } \\ \text { hikari }\end{gathered} 65221 \quad 627$ IR36 $\begin{gathered}\text { Kasa- } \\ \text { lath }\end{gathered} 414 \quad 868$ W107W2002W630W1551W120W149W1718W025W1579b-2W1185W1625 W0002

$\begin{array}{llllllllllllllllllllll}\text { Stowaway Os-1 } & 88.0 & 87.3 & 87.7 & 94.0 & 89.0 & 87.3 & 95.3 & 89.7 & 83.0 & 96.7 & 87.0 & 87.0 & 92.0 & 90.3 & 92.3 & 94.0 & 84.0 & 82.0 & 79.3 & 81.3 & 59.3\end{array}$

$\begin{array}{llllllllllllllllllllll}\text { Tabito II } & 68.7 & 69.0 & 71.0 & 73.0 & 71.3 & 69.0 & 68.3 & 71.7 & 70.0 & 73.0 & 60.0 & 66.3 & 67.7 & 75.3 & 73.3 & 68.7 & 62.3 & 64.0 & 58.0 & 60.3 & 3.3\end{array}$

$\begin{array}{llllllllllllllllllllllll}\text { Onaga I } & 52.0 & 52.7 & 54.7 & 52.3 & 53.3 & 54.3 & 52.7 & 55.7 & 55.7 & 50.7 & 46.3 & 47.3 & 50.3 & 48.3 & 62.0 & 53.3 & 47.0 & 50.7 & 44.7 & 42.7 & 46.0\end{array}$

$\begin{array}{llllllllllllllllllllll}\text { Mashu } & 64.7 & 68.3 & 64.3 & 64.7 & 62.3 & 67.3 & 71.0 & 64.0 & 66.0 & 72.7 & 60.0 & 64.7 & 70.3 & 69.3 & 74.0 & 63.3 & 58.3 & 55.7 & 55.3 & 35.3 & 13.3\end{array}$

1) The numbers are the averages of three trials with different adaptor primers.

2) 21 Oryza strains are listed in Table 1.

other O. rufipogon strains from India or South-East Asian countries are included in the Indica cluster. In addition, an African cultivated rice species, O. glaberrima, made a distinct cluster together with its wild progenitor, O. barthii. Regarding the annual-perennial differentiation in the wild progenitors of Asian cultivated rices, Aggarwal et al. (1999) reported two distinct clusters corresponding to annuals or perennials based on AFLP analyses; however, the present study based on MITE-TDs showed that the W107 (annual) and W120 (perennial) from India are closely related to each other. These results support the idea that the differentiation in the longevity of the Asian wild rices generated responding to ecological properties in wild rice, but not a consequence of speciation (Morishima et al. 1984). To resolve the questions noted above, it will be necessary to examine a large number of strains of wild and cultivated rice species.

MITE-TD analysis has the potential of locating markers throughout Oryza genomes, and of producing detailed phylogenetic trees. This method is also applicable for the isolation of genes tagged with a specific MITE and exploring molecular markers linked with MITEs. Our MITE-TD system generated polymorphic bands between Japonica and Indica at more than twice the frequency of AFLP analysis. All four MITEs used here were very useful for examining 
Table 5. The frequency indexes of polymorphisms between two lines of the nine O.sativa strains detected by the four MITE-TDs ${ }^{1)}$

\begin{tabular}{|c|c|c|c|c|c|c|c|c|c|}
\hline \multirow{2}{*}{ Stowaway Os-1 } & \multicolumn{3}{|c|}{ Japonica } & \multicolumn{2}{|c|}{ Javanica } & \multicolumn{4}{|c|}{ Indica } \\
\hline & A58 & Koshihikari & T65 & 221 & 627 & IR36 & Kasalath & 414 & 868 \\
\hline A58 & & 0.07 & 0.09 & 0.24 & 0.26 & 0.50 & 0.52 & 0.49 & 0.51 \\
\hline Koshihikari & & & 0.04 & 0.21 & 0.24 & 0.48 & 0.50 & 0.48 & 0.50 \\
\hline $\mathrm{T} 65$ & & & & 0.20 & 0.24 & 0.48 & 0.50 & 0.48 & 0.50 \\
\hline 221 & & & & & 0.18 & 0.50 & 0.47 & 0.46 & 0.50 \\
\hline 627 & & & & & & 0.48 & 0.47 & 0.45 & 0.49 \\
\hline IR36 & & & & & & & 0.37 & 0.29 & 0.34 \\
\hline Kasalath & & & & & & & & 0.36 & 0.43 \\
\hline 414 & & & & & & & & & 0.27 \\
\hline \multicolumn{10}{|l|}{868} \\
\hline \multirow{2}{*}{ Tabito II } & \multicolumn{3}{|c|}{ Japonica } & \multicolumn{2}{|c|}{ Javanica } & \multicolumn{4}{|c|}{ Indica } \\
\hline & A58 & Koshihikari & $\mathrm{T} 65$ & 221 & 627 & IR36 & Kasalath & 414 & 868 \\
\hline A58 & & 0.08 & 0.09 & 0.20 & 0.30 & 0.54 & 0.55 & 0.55 & 0.56 \\
\hline Koshihikari & & & 0.05 & 0.21 & 0.29 & 0.54 & 0.55 & 0.53 & 0.55 \\
\hline T65 & & & & 0.19 & 0.28 & 0.53 & 0.56 & 0.54 & 0.54 \\
\hline 221 & & & & & 0.22 & 0.49 & 0.53 & 0.50 & 0.49 \\
\hline 627 & & & & & & 0.51 & 0.53 & 0.50 & 0.51 \\
\hline IR36 & & & & & & & 0.43 & 0.32 & 0.32 \\
\hline Kasalath & & & & & & & & 0.45 & 0.44 \\
\hline 414 & & & & & & & & & 0.24 \\
\hline \multicolumn{10}{|l|}{868} \\
\hline \multirow{2}{*}{ Onaga I } & \multicolumn{3}{|c|}{ Japonica } & \multicolumn{2}{|c|}{ Javanica } & \multicolumn{4}{|c|}{ Indica } \\
\hline & A58 & Koshihikari & $\mathrm{T} 65$ & 221 & 627 & IR36 & Kasalath & 414 & 868 \\
\hline A58 & & 0.04 & 0.06 & 0.21 & 0.22 & 0.48 & 0.50 & 0.52 & 0.46 \\
\hline Koshihikari & & & 0.04 & 0.21 & 0.21 & 0.48 & 0.49 & 0.53 & 0.46 \\
\hline T65 & & & & 0.24 & 0.24 & 0.49 & 0.50 & 0.53 & 0.47 \\
\hline 221 & & & & & 0.18 & 0.44 & 0.43 & 0.46 & 0.39 \\
\hline 627 & & & & & & 0.43 & 0.41 & 0.44 & 0.41 \\
\hline IR36 & & & & & & & 0.43 & 0.28 & 0.25 \\
\hline Kasalath & & & & & & & & 0.36 & 0.36 \\
\hline 414 & & & & & & & & & 0.27 \\
\hline \multicolumn{10}{|l|}{868} \\
\hline \multirow{2}{*}{ Mashu } & \multicolumn{3}{|c|}{ Japonica } & \multicolumn{2}{|c|}{ Javanica } & \multicolumn{4}{|c|}{ Indica } \\
\hline & A58 & Koshihikari & T65 & 221 & 627 & IR36 & Kasalath & 414 & 868 \\
\hline A58 & & 0.14 & 0.11 & 0.40 & 0.41 & 0.70 & 0.68 & 0.72 & 0.72 \\
\hline Koshihikari & & & 0.15 & 0.43 & 0.43 & 0.71 & 0.70 & 0.73 & 0.73 \\
\hline $\mathrm{T} 65$ & & & & 0.42 & 0.40 & 0.71 & 0.69 & 0.72 & 0.72 \\
\hline 221 & & & & & 0.47 & 0.70 & 0.67 & 0.73 & 0.72 \\
\hline 627 & & & & & & 0.64 & 0.65 & 0.65 & 0.64 \\
\hline IR36 & & & & & & & 0.56 & 0.42 & 0.46 \\
\hline Kasalath & & & & & & & & 0.56 & 0.60 \\
\hline 414 & & & & & & & & & 0.35 \\
\hline 868 & & & & & & & & & \\
\hline
\end{tabular}

1) The frequency index was calculated as 1-SC ( $\mathrm{SC}$ is described in Materials and Methods).

the genetic diversity among closely related species such as the AA-genome species. For distantly related species such as the AA- and CC-genomes, however, this method is not suitable because the distribution of MITEs differs in them. Among the MITEs examined here, Mashu could most efficiently detect polymorphisms for the AA-genome species. We could not conclude whether Mashu has stabilized or still remains active. Even if Mashu is inactive, it is a powerful MITE system for providing molecular markers throughout the rice genome for TD analysis.

\section{Acknowledgements}

This work was supported by grants from the Ministry of Education, Science and Culture, Japan, and the Rice Genome Research Program (RGP). 
Stowaway Os -1

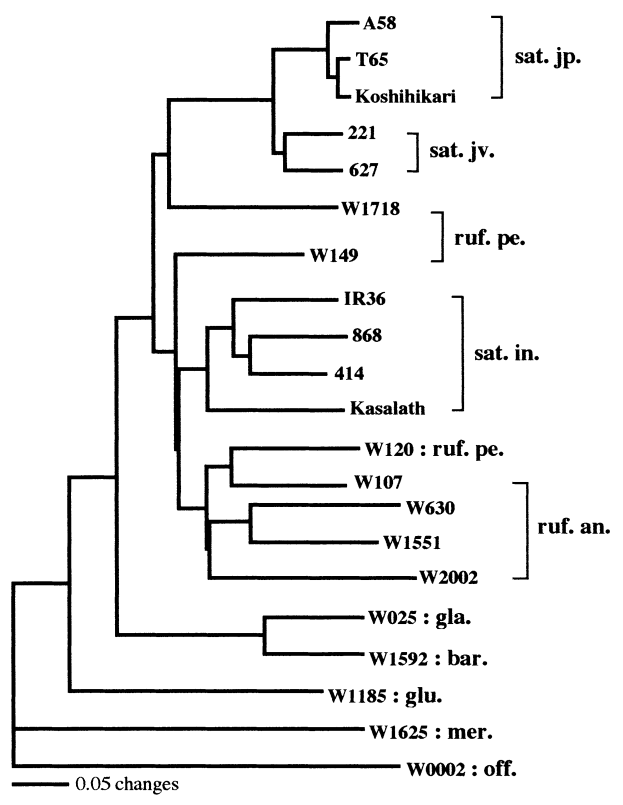

Onaga I

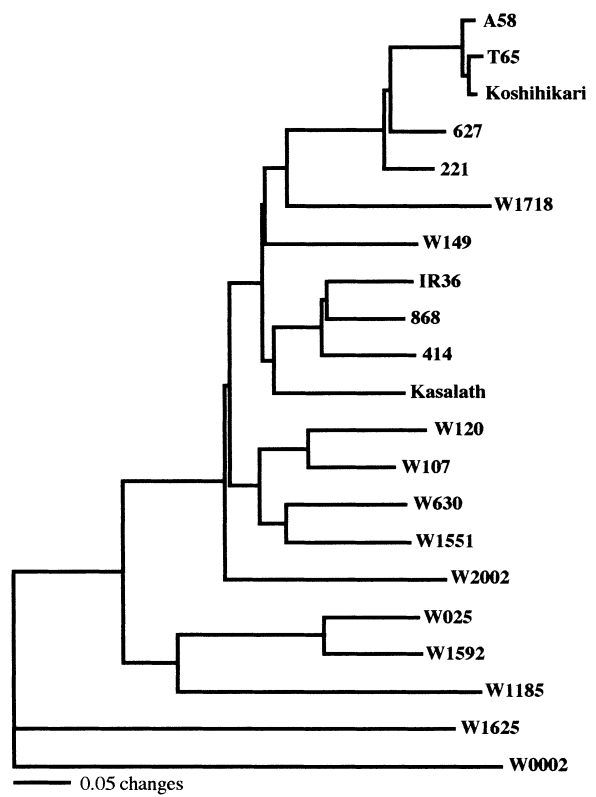

Tabito II

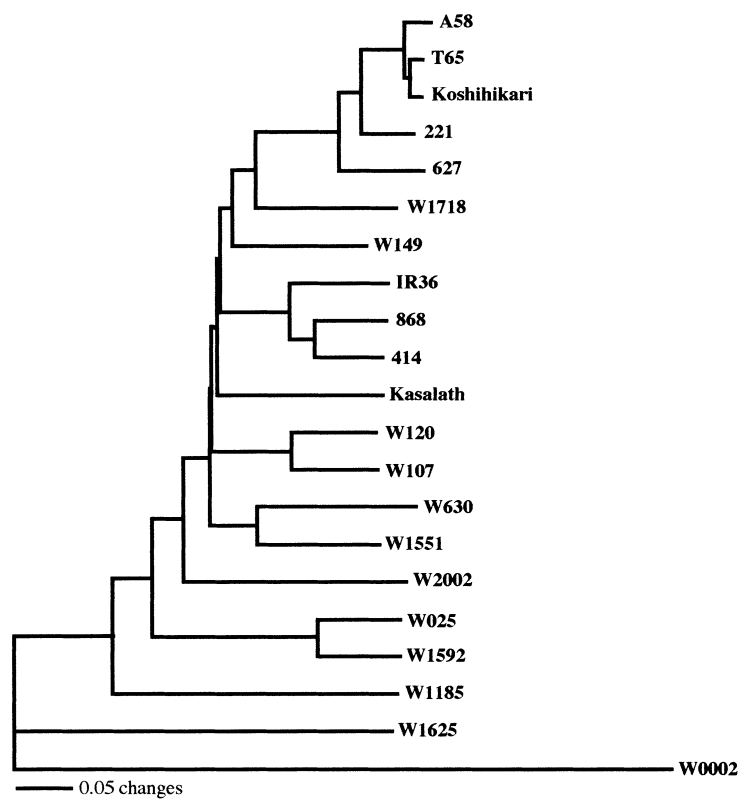

Mashu

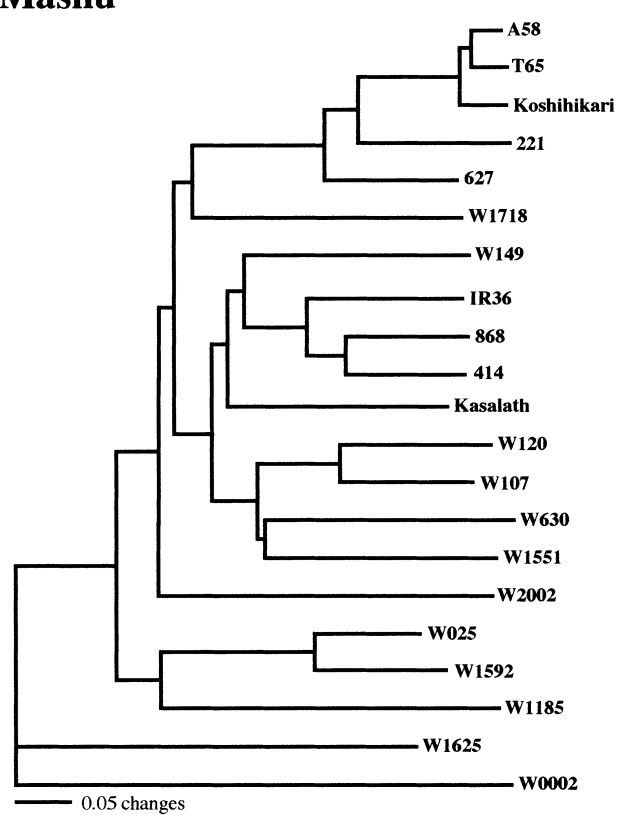

Fig. 2. Phylogenetic trees of the 21 strains of Oryza species constructed with individual MITE-TDs.

Dendrograms were drawn with the NJ methods based on polymorphisms obtained by MITE-TDs. The root length, which reflects the genetic distance, is equivalent for all the trees. The abbreviations for species names and types are as follows; sat: sativa, ruf: rufipogon, gla: glaberrima, bar: barthii, glu: glumaepatula, mer: meridionalis, off: officinalis, jp: Japonica, jv: Javanica, in: Indica, an: annual, pe: perennial.

\section{Literature Cited}

Aggarwal,R.K., D.S.Brar, S.Nandi, N.Huang and G.S.Khush (1999) Phylogenetic relationships among Oryza species revealed by AFLP markers. Theor. Appl. Genet. 98: 1320-1328.

Bennetzen,J.L. (2000) Transposable element contributions to plant gene and genome evolution. Plant Mol. Biol. 42: 251-269.

Bennetzen,J.L., P.SanMiguel, M.S.Chen, A.Tikhonov, M.Francki and Z.Avramova (1998) Grass genomes. Proc. Natl. Acad. Sci.
USA 95: 1975-1978.

Bureau,T.E. and S.R.Wessler (1994a) Stowaway: a new family of inverted repeat elements associated with the genes of both monocotyledonous and dicotyledonous plants. Plant Cell 6: 907-916. Bureau,T.E. and S.R.Wessler (1994b) Mobile inverted-repeat elements of the Tourist family are associated with the genes of many cereal grasses. Proc. Natl. Acad. Sci. USA 91: 14111415.

Bureau,T.E., P.C.Ronald and S.R.Wessler (1996) A computer-based 
systematic survey reveals the predominance of small invertedrepeat elements in wild-type rice genes. Proc. Natl. Acad. Sci. USA 93: 8524-8529.

Casa,A.M., C.Brouwer, A.Nagel, L.Wang, Q.Zhang, S.Kresovich and S.R.Wessler (2000) The MITE family heartbreaker (Hbr): molecular markers in maize. Proc. Natl. Acad. Sci. USA 97: 10083-10089.

Dice,L.R. (1945) Measures of the amount of ecological association between species. Ecology 26: 297-302.

Dubcovsky,J., W.Ramakrishna, P.J.SanMiguel, C.S.Busso, L.L.Yan, B.A.Shiloff and J.L.Bennetzen (2001) Comparative sequence analysis of colinear barley and rice bacterial artificial chromosomes. Plant Physiol. 125: 1342-1353.

Feschotte, C., N.Jiang and S.R. Wessler (2002) Plant transposable elements: where genetics meets genomics. Nat. Rev. Genet. 3: 329-341.

Jiang,N. and S.R. Wessler (2001) Insertion preference of maize and rice miniature inverted repeat transposable elements as revealed by the analysis of nested elements. Plant Cell 13: 25532564.

Kumar,A. and J.L.Bennetzen (1999) Plant retrotransposons. Annu. Rev. Genet. 33: 479-532.

Lisch,D.R., M.Freeling, R.J.Langham and M.Y.Choy (2001) Mutator transposase is widespread in the grasses. Plant Physiol. 125: 1293-1303.

Lynch,M. (1990) The similarity index and DNA fingerprinting. Mol. Biol. Evol. 7: 478-484.

Maheswaran,M., P.K.Subudhi, S.Nandi, J.C.Xu, A.Parco, D.C.Yang and N.Huang (1997) Polymorphism, distribution, and segregation of AFLP markers in a doubled haploid rice population. Theor. Appl. Genet. 94: 39-45.

Morishima,H., Y.Sano and H.I.Oka (1984) Differentiation of perennial and annual types due to habitat conditions in the wild rice Oryza perennis. Plant Syst. Evol. 144: 119-135.

Nagano,H., S.Kawasaki, Y.Kishima and Y.Sano (2000) Structural differences in the vicinity of the waxy locus among the Oryza species with the AA-genome: identification of variable regions. Theor. Appl. Genet. 100: 376-383.

Nagano,H., M.Kunii, T.Azuma, Y.Kishima and Y.Sano (2002) Characterization of the repetitive sequences in a $200-\mathrm{kb}$ region around the rice waxy locus: diversity of transposable elements and presence of veiled repetitive sequences. Genes Genet. Syst. 77: 69-79.

Nagano,H., L.Wu, S.Kawasaki, Y.Kishima and Y.Sano (1999) Genomic organization of the $260 \mathrm{~kb}$ surrounding the waxy locus in a Japonica rice. Genome 42: 1121-1126.

Saitou,N. and M.Nei (1987) The neighbor-joining method: a new method for reconstructing phylogenetic trees. Mol. Biol. Evol. 4: 406-425.

SanMiguel,P., A.Tikhonov, Y.K.Jin, N.Motchoulskaia, D.Zakharov, A. MelakeBerhan, P.S. Springer, K.J. Edwards, M. Lee, Z.Avramova and J.L.Bennetzen (1996) Nested retrotransposons in the intergenic regions of the maize genome. Science 274: 765-768

Suoniemi, A., K. AnamthawatJonsson, T.Arna and A.H.Schulman (1996) Retrotransposon BARE-1 is a major, dispersed component of the barley (Hordeum vulgare L) genome. Plant Mol. Biol. 30: 1321-1329.

Swofford,D.L. (1998) PAUP*. Phylogenetic Analysis Using Parsimony (*and Other Method), Version 4. Sinauer Association, Sunderland, Massachusetts.

Tarchini,R., P.Biddle, R.Wineland, S.Tingey and A.Rafalski (2000) The complete sequence of $340 \mathrm{~kb}$ of DNA around the rice Adh1-adh2 region reveals interrupted colinearity with maize chromosome 4. Plant Cell 12: 381-391.

Turcotte,K., S.Srinivasan and T.Bureau (2001) Survey of transposable elements from rice genomic sequences. Plant J. 25: 169-179.

Van den Broeck, D., T. Maes, M.Sauer, J.Zethof, P.De Keukeleire, M.D'Hauw, M.Van Montagu and T.Gerats (1998) Transposon display identifies individual transposable elements in high copy number lines. Plant J. 13: 121-129.

Wessler, S.R., T.E. Bureau and S.E. White (1995) LTR-retrotransposons and MITEs: important players in the evolution of plant genomes. Curr. Opin. Genet. Dev. 5: 814-821.

Zhou,Z. and J.P.Gustafson (1995) Genetic-variation detected by DNAfingerprinting with a rice minisatellite probe in Oryza sativa L. Theor. Appl. Genet. 91: 481-488.

Zhu,J., M.D.Gale, S.Quarrie, M.T.Jackson and G.J.Bryan (1998) AFLP markers for the study of rice biodiversity. Theor. Appl. Genet. 96: 602-611. 